Prehospital care

\section{Prehospital care in the EMJ}

\section{R Mackenzie, C Laird}

\section{Developments in the prehospital care content of the EMJ}

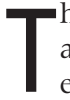
here is increasing recognition and acceptance of the view that the emergency medical system should provide seamless and appropriate care from the moment the patient is injured, taken ill, or seeks help. Coupled with this is a developing understanding that the many different demands for emergency or "unscheduled" health care can only be met by new and innovative working patterns and practices within emergency departments, ambulance services, and primary healthcare teams. The work on ambulance service roles and curriculums and the evolving emergency care practitioner concept published in this edition are just two examples of work being undertaken to meet prehospital care demands and improve access. This work is important and necessary. Among all this activity however, there is a small but real risk of neglecting the educational needs of those who currently provide urgent immediate care needs in the prehospital environment.

So how can the EMJ help? Firstly, our $\mathrm{ABC}$ of Community Emergency Care is aimed at both existing prehospital practitioners and those who are part of evolving systems of care. We hope that it provides a starting point for further educational and curriculum development and we would welcome your input and feedback. Secondly, the EMJ is also the official journal of the British Association for Immediate Care (BASICS) and the Faculty of Pre-hospital Care of the Royal College of Surgeons of Edinburgh. Both organisations have a great deal of expertise and experience within their memberships and this should be shared. Consider the use of ketamine for example. Most experienced immediate care doctors consider that there is no real alternative drug when faced with a trapped, conscious patient who requires pharmacological sedation and analgesia to facilitate extrication and rescue. ${ }^{12}$ Yet there are few trials to support its safety or effectiveness in prehospital care and a lively debate continues regarding its use in the emergency department. The summary of product characteristics for ketamine states that it should be used "only in hospitals by or under the supervision of experienced medically qualified anaesthetists except under emergency conditions". ${ }^{3}$ Serious injury with entrapment is clearly such an emergency condition. The real question is one of patient safety. Keith Porter's personal audit of prehospital ketamine use is a good example of how safe practice can be shared. ${ }^{4}$ Although we encourage submission of similar audit and review, the sharp contrast in the data and level of evidence available for emergency department use compared with prehospital use is striking and further coordinated primary research is essential. Submitting such research to the $E M J$ is one of the best ways to ensure that it is disseminated widely to those who need to know.

Thirdly, many of the prehospital readership have commented that there is little guidance on equipment selection for prehospital and emergency department use despite the fact that equipment considerations can have a major impact on our ability to provide care. Take for example the apparently simple intervention of intraosseous access. What devices are available and what evidence is there of any advantage or disadvantage in the prehospital or emergency department setting? Although most Best Evidence Topic reports (BETs) and clinical topic reviews focus on the evidence underpinning diagnostic strategies or therapeutic interventions, there is a need to disseminate answers to equipment questions such as these. The well established structure of the BET (asking the right question, searching for the evidence, appraising the evidence, and providing a summary relevant to your practice $)^{5}$ is just as relevant to medical devices and novel equipments as it is to pharmaceuticals and diagnostic tests. The evidence may however be harder to find given that published clinical trials are not necessarily the place to find the rationale for an equipment development. We welcome proposals for these Best Evidence Equipment Reviews and hope to publish some examples in the near future.

Finally, case reports may not be evidence based but they certainly have a place in education, training, and informing practice. Much adult learning is experiential and we should not dismiss the opportunity to learn from others. Although we encourage the submission of case reports relevant to prehospital care and the prehospital/ emergency department interface, our experience is that they are of little educational value if they are not accompanied by a brief but comprehensive review of the relevant literature and, where possible, commentary from experienced practitioners and experts in the field. We therefore invite authors to contact us and discuss their case reports before submission so that we may provide editorial guidance and commission suitable commentary.

These additional developments in the prehospital care content of the EMJ represent a strong editorial commitment to providing evidence, education, commentary, experience, and debate throughout the entire patient journey. We hope that they will complement the existing content and help provide for the educational needs of the prehospital and emergency department readership.

Emerg Med J 2004;21:270.

doi: $10.1136 / \mathrm{emj} .2004 .014951$

\section{Authors' affiliations}

R Mackenzie, C Laird, Associate Editors, Prehospital Care

Correspondence to: $\operatorname{Dr} \mathrm{R}$ Mackenzie; Rod@magpas.org.uk

\section{REFERENCES}

1 Cottingham R, Thompson K. Use of ketamine in prolonged entrapment. J Accid Emerg Med 1994; 11:189-91.

2 Mackenzie R. Pre-hospital care: analgesia and sedation. J R Army Med Corps 2000;146:117-27.

3 Electronic Medicines Compendium. Ketamine. Summary of product characteristics. http:// emc.medicines.org.uk (accessed 30 Jan 2004).

4 Porter K. Ketamine in prehospital care. Emeg Med J 2004:21:351-4.

5 Carley SD, Mackway-Jones K, Jones A, et al Moving towards evidence based emergency medicine: use of a structured critical appraisal journal club. J Accid Emerg Med 1998;15:220-2 


\section{Ketamine is a safe, effective, and appropriate technique for emergency department paediatric procedural sedation}

\section{S M Green, B Krauss}

\section{Ketamine has an important role in the management of acutely injured children}

$\square$ hree reports in this issue of the journal substantively advance the status of ketamine for paediatric procedural sedation in the emergency department (ED). McGlone et $a l^{1}$ and Ellis $e t$ al $^{2}$ report a total of 590 ketamine administrations, together noting a high level of sedation efficacy, strong degrees of parental and staff satisfaction, and an adverse effect profile readily manageable by trained emergency physicians. In a third report, Howes $^{3}$ capably reviews the now abundant literature supporting the safety of this dissociative sedative technique.

These reports are entirely consistent with many previous ED series from the United Kingdom ${ }^{4-7}$ and elsewhere ${ }^{8-18}$ reporting the safety of ketamine in literally thousands of children. The exceptional track record for this drug in various non-ED settings has also been well reported. ${ }^{19}$

Ketamine is now widely accepted as a standard of care for ED paediatric procedural sedation in the United States. ${ }^{8-22}$ The unique dissociative state induced by ketamine is inconsistent with all accepted definitions of general anaesthesia, and thus any allegation that this drug is an "anaesthetic" —and thus somehow dangerous-is an exercise in semantics rather than in safety or appropriateness. $^{21}$ The Joint Commission on Accreditation of Healthcare Organizations (JCAHO) - the United States accrediting body for hospitalshas confirmed that ED ketamine administration is fully compliant with their standards when administered according to protocol. ${ }^{22}$ The Executive Committee of the American Academy of Pediatrics' Section on Anesthesiology has acknowledged that "ketamine has had wide and safe use by nonanesthesiologists", ${ }^{23}$ and perhaps no non-anaesthetists are better trained to manage sedation and its complications than are emergency physicians given their skill set. Resistance from American chiefs of anaesthesia to
ED ketamine protocols-common in the 1990s-has now all but evaporated attributable in large part to the robust data showing the safety and efficacy of ketamine use for children by emergency physicians.

Yes, ketamine is extremely safe. However, its administration requires skilled practitioners well versed in its unique action, aware of its many contraindications, and fully prepared to manage its potential complications with confidence. Airway malalignment (0.7\% incidence), laryngospasm $(0.4 \%)$, and apnea/respiratory depression $(0.3 \%)$ do rarely occur in the ED setting, however no adverse outcomes relating to these complications have been reported. ${ }^{1-18}$ In 33 years of regular use there have been no reported reports of ketamine associated clinically significant aspiration in patients without established contraindications. ${ }^{18-20}$ Indeed, the unique retention of protective airway reflexes with ketamine despite full dissociation makes this drug especially well suited for urgent and emergent procedures commonly performed in the ED on non-fasted patients. ${ }^{24}$

ED use of ketamine should optimally occur only in strict accordance with a protocol such as that sanctioned by the JCAHO. ${ }^{22}$ Ketamine is not the perfect ED sedative, as emesis well into recovery is not uncommon $(6.7 \% \text { incidence })^{8}$ and recovery times (median 110 minutes) are substantially longer than with newer ultra-short acting agents such as propofol..$^{20}$

If ketamine is not already in widespread use in EDs in the United Kingdom, it is not because the drug is unsafe, ineffective, or that its experience has not been rigorously reported. It is probably because of the qualifications of the practitioners to whom responsibility for its management would rest. Ketamine is not an appropriate agent for unsupervised use by junior house officers. In the United States ketamine is administered by senior registrar or consultant level career emergency physicians (or by trainees under their direct supervision) who supervise the ED 24 hours per day and are immediately available to manage potential sedation complications. American emergency physicians undergo at least three years of postgraduate training in emergency medicine, including expert level skills in procedural sedation and its component skills: advanced airway management, vascular access, pharmacology, and resuscitation.

Emergency physicians in the United Kingdom who possess the above requisites should not be hindered from enacting ketamine protocols and administering this drug to select patients. Indeed it would seem the duty of anaesthetists and hospital leadership to-whenever and however appropriate-assist emergency physicians in providing such state of the art management of procedural pain and anxiety for the benefit of the acutely injured children we serve.

Emerg Med J 2004;21:271-272.

doi: $10.1136 / \mathrm{emi} .2004 .015370$

\section{Authors' affiliations}

S M Green, Department of Emergency Medicine, Loma Linda University Medical Center and Children's Hospital, Loma Linda, California, USA

B Krauss, The Division of Emergency Medicine, Children's Hospital and Harvard Medical School, Boston, Massachusetts, USA

Correspondence to: Dr S M Green, Loma Linda University Medical Center A-108, 11234 Anderson Street, Loma Linda, CA 92354, USA; stevegreen@tarascon.com

\section{REFERENCES}

1 McGlone RG, Howes MC, Joshi M. The Lancaster experience of 2.0 to $2.5 \mathrm{mg} / \mathrm{kg}$ intramuscular ketamine for paediatric sedation: 501 cases and analysis. Emerg Med J 2004;21:290-5.

2 Ellis DY, Husain HM, Saetta JP, et al. Procedural sedation for paediatric patients: a prospective audit of ketamine use in the emergency department. Emerg Med J 2004:21:286-9.

3 Howes MC. Ketamine for paediatric sedation/ analgesia in the emergency department. Emerg Med J 2004;21:275-80.

4 McGlone RG, Ranasinghe S, Durham S. An alternative to "brutacaine": a comparison of low dose intramuscular ketamine with intranasal midazolam in children before suturing. J Accid Emerg Med 1998;15:231-6.

5 Holloway VJ, Husain HM, Saetta JP, et al Accident and emergency department led implementation of ketamine sedation in paediatric practice and parental response. J Accid Emerg Med 2000;17:25-8.

6 Ackworth JP, Purdie D, Clark RC. Intravenous ketamine plus midazolam is superior to intranasal midazolam for emergency paediatric procedural sedation. Emerg Med J 2001;18:39-45.

7 McGlone RG, Fleet T, Durham S, et al. A comparison of intramuscular ketamine with high dose intramuscular midazolam with and without intranasal flumazenil in children before suturing. Emerg Med J 2001;18:34-8. 
8 Green SM, Rothrock SG, Lynch EL, et al. Intramuscular ketamine for pediatric sedation in the emergency department: safety profile with 1,022 cases. Ann Emerg Med 1998:31:688-97.

9 Green SM, Rothrock SG, Harris T, et al. Intravenous ketamine for pediatric sedation in the emergency department: safety profile with 156 cases. Acad Emerg Med 1998;5:971-6.

10 Pena BMG, Krauss B. Adverse events of procedural sedation and analgesia in a pediatric emergency department. Ann Emerg Med 1999:34:483-90.

11 Sherwin TS, Green SM, Khan A, et al. Does adjunctive midazolam reduce recov-ery agitation after ketamine sedation for pediatric procedures? A randomized, double-blind, placebo-con-trolled trial. Ann Emerg Med 2000;35:239-44.

12 Wathen JE, Roback MG, Mackenzie T, et al. Does midazolam alter the clinical effects of intravenous ketamine sedation in children? A double-blind, randomized, controlled emergency department trial. Ann Emerg Med 2000;36:579-88.
$13 \mathrm{Ng} \mathrm{KC}$, Ang SY. Sedation with ketamine for paediatric procedures in the emergency department-a review of 500 cases. Singapore Med J 2002;43:300-4

14 Ip U, Saincher A. Safety of pediatric procedural sedation in a Canadian emergency department. Can J Emerg Med 2000;2:15-20.

15 McCarty EC, Mencio GA, Walker A, et al. Ketamine sedation for the reduction of children's fractures in the emergency department. J Bone Joint Surg 2000;7:912-18.

16 Petrack EM, Marx CM, Wright MS. Intramuscular ketamine is superior to meperidine, promethazine, and chlorpromazine for pediatric emergency department sedation. Arch Pediatr Adolesc Med 1996;150:676-81.

17 Pruitt JW, Goldwasser MS, Sabol SR, et al. Intramuscular ketamine, midazolam, and glycopyrrolate for pediatric sedation in the emergency department. J Oral Maxillofac Surg 1995;53:13-17.

18 Green SM, Kuppermann N, Rothrock SG, et al. Predictors of adverse events with ketamine sedation in children. Ann Emerg Med 2000;35:35-42.

19 Green SM, Johnson NE. Ketamine sedation for pediatric procedures: Part 2, Review and implications. Ann Emerg Med 1990; 19: 1033-46.

20 Krauss B, Green SM. Sedation and analgesia for procedures in children. N Engl J Med 2000:342:938-45.

21 Green SM, Krauss B. The semantics of ketamine. Ann Emerg Med 2000;36:480-2.

22 JCAHO. Care of patients: examples of compliance. Oakbrook Terrace, IL: Joint Com-mis-sion on Accreditation of Healthcare Organizations, 1999:87-91.

23 Means LJ, Ferrari L, Mancuso TJ, et al. The pediatric sedation unit: a mechanism for safe pediatric sedation. [Letter]. Pediatrics 1999; 103:199-201

24 Green SM, Krauss B. Pulmonary aspiration risk during $E D$ procedural sedation - an examination of the role of fasting and sedation depth. Acad Emerg Med 2002;9:35-42.

\section{Ketamine is not a safe, effective, and appropriate technique for emergency department paediatric procedural sedation}

\section{N S Morton}

\section{Definitive studies are needed}

lit n the United Kingdom, there is very ittle formal competency based training in the techniques of paediatric procedural sedation except in the specialty of dentistry. ${ }^{12}$ Procedural sedation is often delegated to junior medical staff using a local protocol. The procedures for informed consent, documentation, monitoring, recovery, follow up, and audit are varied and often non-existent. This situation is changing as new clinical governance and training systems are put in place. In Scotland, the Scottish Intercollegiate Guidelines Network (SIGN) has produced a multidisciplinary, evidence based, peer reviewed guideline for the management of paediatric procedural sedation. ${ }^{3}$ The consensus view of this group was that, in Scotland, ketamine (and agents such as propofol, fentanyl, alfentanil, and remifentanil) should only be used by those trained in and with adequate ongoing experience of paediatric anaesthesia and/or intensive care. It should only be used in a hospital setting. The product licence (and indeed the vial) states that ketamine is an intravenous general anaesthetic agent that should only be used by a trained

The specialty of accident and emergency medicine is developing rapidly in the UK and consultant led and consultant based 24 hour services are becoming more common. The Faculty of Accident and Emergency Medicine were formally involved in the development and peer review of the SIGN guidelines and were represented on the working group by one of the few specialist paediatric A\&E consultants in the UK. However, some members of that Faculty feel they were not adequately consulted, that their practice was being undermined, and that the guidelines apply only in Scotland. SIGN guidelines are living documents and can be changed in the light of new evidence. The National Audit of paediatric ketamine use in emergency departments in the UK mentioned in this issue is welcome and any further research on the utility of ketamine when given by non-anaesthetists in the UK hospital setting is also to be welcomed.

Before this technique can be widely supported for use in paediatric A\&E anaesthetist. practice in the UK several issues need to be addressed. The most important provision is adequate training and on-going experience in paediatric sedation and analgesic techniques. Better communication and links between specialties that have to deal with children would also be of great benefit. The Academy of Royal Colleges in the UK has observed guidelines for safe sedation were not being implemented and proposed some actions to improve adherence to good practice. ${ }^{4}$ Their advice was that every hospital should have a multidisciplinary sedation group led jointly by an anaesthetist and another specialist. They should monitor sedation activity, assess the need for sedation, oversee the local implementation of national guidelines, and audit the safety and adverse effects of sedation practices in their unit. Where children are involved, paediatric specialist expertise and advice should form part of these groups. In many hospitals these recommendations have still not been implemented and dialogue between specialties such as A\&E and anaesthesia in some hospitals can be zero!

Children and families must be given informed choices based on clear information on risks and benefits, efficacy, and safety. If you are going to promote widespread adoption of a technique with a questionable adverse effect profile in children, you need to be sure that adequate controlled comparisons have been made of the alternatives. For ketamine, the definitive controlled and randomised studies compared with modern general anaesthesia have yet to be carried out in an A\&E setting. In paediatric radiology general anaesthesia has recently been shown to be safer, more reliable, more efficient, and more cost effective than sedation of children for MRI scans. ${ }^{5}$ Until proper comparative 
studies are done, I think the jury is out despite arguments in favour of ketamine presented in this issue.

We do have a duty to collaborate and cooperate across the specialties to produce quality evidence. One benefit of this is to gain an understanding of what different specialties regard as a standard of care. A recent study in Dartmouth USA where procedural sedation undertaken by non-anaesthetists was videotaped and reviewed by an independent panel revealed major problems with the perceptions of those carrying out the procedures as to the efficacy and safety of their technique. ${ }^{6}$ I hope such projects and this type of debate will help promote cross specialty understanding and collaboration in the best interests of children.

Emerg Med J 2004;21:272-273.

doi: 10.1136/emi.2003.011411

Correspondence to: N S Morton, Department of Paediatric Anaesthesia, Intensive Care and Pain Management, Royal Hospital for Sick Children, Glasgow G3 8SJ, UK; Neil.Morton@yorkhill. scot.nhs.uk

The author is Chairman of the SIGN Development Group for SIGN Guideline 58 www. sign.ac.uk

\section{REFERENCES}

1 Department of Health. An independent expert working group. Standards in conscious sedation for dentistry, 2000. http://dstg.co.uk/teaching/ oct2000sicsfd/octprint.htm

2 Chief Medical Offcer/Chief Dental Officer Report by a group chaired by the $\mathrm{CMO}$ and the CDO: a conscious decision. A review of the use of general anaesthesia and conscious sedation in primary dental care. London: Department of Health 2000

3 Scottish Intercollegiate Guidelines Network. SIGN guideline 58. Safe dedation of children undergoing diagnostic and therapeutic procedures. (http://www.sign.ac.uk).

4 Academy of Medical Royal Colleges. Implementing and ensuring safe sedation practice for healthcare procedures in adults 2001 ; (http:// www.aomrc.ac.uk).

5 Malviya S, Voepel-Lewis T, Eldevik OP, et al. Sedation and general anaesthesia in children undergoing MRI and CT: adverse events and outcomes. Br J Anaesth 2000;84:743-8.

6 Society for Pediatric Anesthesia. Newslette 2003; (http://www.pedsanesthesia.org/ newsletter/spring2003/03_mtg_review.shtml).

\section{Emergency care practitioners}

\section{J Scott, C Carney}

\section{Important developments in the delivery of emergency care}

W ith rapidly changing healthcare provision, many new job roles and titles are appearing in emergency, primary, and secondary care. This proliferation has led to a degree of confusion that may have detracted from some very important developments that are taking place in the delivery of out of hospital emergency and unscheduled (urgent) care.

In out of hospital care, the previous descriptors of technician, paramedic, and immediate care doctor were born out of a hierarchic structure, however as education and training opportunities have occurred there has been a blurring of the edges between all these groups.

Within primary care and A\&E units, the introduction of new targets and ways of working are demanding the introduction of clinicians with new skills and competencies.

In tracing the origins of these new developments, the first ideas on practitioner development in prehospital care were put forward by Douglas Chamberlain, the then Chairman of the Joint Royal Colleges Ambulance Liaison Committee (JRCALC) and the Ambulance Services Association (ASA) in January 2000. ${ }^{1}$ The resulting "practitioner in emergency care" (PEC) concept started the debate that has evolved or metamorphosed to become the more elegant sounding emergency care practitioner (ECP).

What does this title mean and what areas of practice may those in these new roles undertake clinically? There is no doubt that when the Changing Working Force (NHS Modernisation Agency) programme ${ }^{2}$ proposed the extension of the work being undertaken in Coventry and Warwickshire to develop professionals from a paramedic and nursing background to dual role, its emphasis was on reducing the A\&E four hour waiting times.

There are other new pressing demands on out of hospital emergency care provision. The new GP contract and the change in the provision of out of hours, the incessant and increasing demand upon ambulance trusts through the 999 service, and this year the increasing threat posed to round the clock hospital emergency services under the working time directive, are all leading to the requirement for clinicians to be trained to undertake new roles.

One such educational package with a clear view to the end clinical requirements is described in this issue. ${ }^{3}$

The dangers are great. Merely to train in new areas and increase salaries of these new roles does nothing to increase the number of staff in the system nor does it encourage new ways of working or providing health care.

The underpinning need is to invoke fundamental system change in emergency and urgent care and new roles are merely a component. If we take the opportunity to develop staff (career progression) and at the same time create an integrated and clinically supported system, then patient care can be significantly improved while minimising risk associated with uncoordinated development of "ad hoc" new roles, sometimes in reaction to changes in healthcare circumstances.

There are some ideological debates to be concluded. We want to break down barriers between professional groups and in doing so create functional teams; supportive of each other but able to deal with the patient's problem at the first point of contact. "Appropriate care at the point of need" (ACAPON) is a concept developed in a practice in Norfolk using a community paramedic and creating a team from differing professional backgrounds to improve each other's competencies and abilities to manage the problem presented by the patient in the community. The initial pilot, which has been reported in the Health Service Journal, ${ }^{4}$ showed considerable benefit in preventing patients being transferred to the local district general hospital (some 20 miles away) in 30\% of patients seen by the community paramedic.

People within such a system require senior clinical support (whichever way round that system triangle is createdpyramid or inverted pyramid). While at the point of contact, the staff member is acting independently their actions are not truly autonomous; but constrained 
and supported by the system of care provided by the team. This fundamental point about defining the system the practitioner is about to work within is central to considering the person's task and therefore the educational or curricular content and planning of courses.

The ECP has to be able to undertake the "first point contact" role and to achieve this, the person must be able to undertake and interpret the findings gained during a formal history taking and comprehensive and appropriate examination. However, the system will have gained little if at that point the patient is merely referred to some other clinician or agency. The ECP has to be able to start simple investigations and to provide explanation to the patient together with starting a treatment management plan, some of which the ECP must be able to provide.

From this comparatively simple description I suspect many different perceptions of this new person will have been acquired and they are probably all correct. However, go and challenge your vision again, but this time look for the reasons why the virtual image may fade as we turn it into reality.

Problems with implementing this new practitioner most effectively still remain, drug provision being the most obvious, but none are insurmountable.

It is the end product that must be clearly defined. Educational pathways must be refined to support these developments and must link to core programmes. There is a chance here to promote the undergraduate (before registration) and postgraduate (after registration) components of a career pathway. This would enable those who wish to work at some point in their clinically active life in the out of hospital or in-hospital fields to rotate into different roles with only minor additional development in modular form from a common core knowledge set acquired in basic ECP training. This would have clear service and individual benefits compared with the current more secular training courses.

Finally, how will success be recognised or gauged? No single organisation will be able, except in very parochial terms, to evaluate this new role. We will require central funding and academic university department assistance to undertake this evaluation work. But it will have to include scrutiny of the ECP educational development, the patients' perspective, the ECP's effect on the whole system of health delivery-both primary and secondary-and the benefits accrued by the individual clinicians involved.

Emerg Med J 2004;21:273-274.

doi: $10.1136 / \mathrm{emj} .2004 .014381$

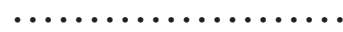

Authors' affiliations

J Scott, C Carney, East Anglian Ambulance

NHS Trust, Norwich, Norfolk, UK

Correspondence to: Dr J Scott, Ambulance Headquarters, East Anglian Ambulance NHS Trust, Hellesdon, Norwich, Norfolk NR6 5NA, UK; john.scot!@eaamb.nhs.uk

\section{REFERENCES}

1 Joint Royal Colleges and Ambulance Liaison Committee and the Ambulance Services Association. The Future role and education of paramedic ambulance service personnel, emerging concepts. London: A subcommittee of the Joint Royal Colleges and Ambulance Liaison Committee and the Ambulance Services Association, January 2000.

2 Whintle C. The NHS change workforce programme national course material. Norwich: Stationery Office, 2003.

3 Doy R, Turner K. The giraffe: the emergency care practitioner; Fit for purpose? The East Anglian experience. Emerg Med J 2004;21:365-6.

4 Everden P, Eardley M, Lorgelly P, et al. Change of pace. The ACAPON Model. Health Service Journal 2003; 113:28-30. 